\title{
HMXBs in the Galaxy and the MCs
}

\author{
Q. Z. Liu \\ Purple Mountain Observatory, Academia Sinica, Nanjing 210008, China \\ email: qzliu@pmo.ac.cn
}

\begin{abstract}
The population of HMXBs in the MCs and the Galaxy is investigated. The SMC has an over-abundant population of HMXBs. This provides the clues that the SMC should be more active in massive star formation. The pulse periods in the SMC are slightly shorter than those in the Galaxy, implying that most of the pulsars in Be/X-ray binaries are not spin-up.
\end{abstract}

Keywords. Massive X-ray binaries, the Galaxy, the Magellanic Clouds

\section{Introduction}

High-mass X-ray binaries (HMXBs) consist of a neutron star or a black hole, orbiting a massive star. The X-ray emission in these sources is due to accretion of matter from the early-type star by the compact companion. Conventionally HMXBs can be further divided into two subgroups: those in which the primary is a Be star and those in which the primary is a supergiant.

\section{HMXB populations in the MCs and the Galaxy}

$\mathrm{X}$-ray observations have revealed that the MCs contain an unexpectedly large number of HMXBs, especially in the SMC. Up to now, 92 known or probable HMXB sources in the SMC and 36 HMXBs in the LMC have been identified (Liu et al. 2005) and they continue to be discovered, although only a small fraction of these are active at any one time because of their transient nature. Of the $128 \mathrm{HMXBs}, 4$ of the objects are supergiant systems, and all the rest are likely Be/X-ray binaries. To compare the HMXB populations in the MCs and in our Galaxy, we collected the HMXBs in the three galaxies from our previous X-ray binary catalogues (Liu et al. 2000, 2005) and recent literature.

Table 1 summarizes the HMXB populations (and the LMXB populations as well (Liu et al. 2001 and the updated version)) in the three galaxies. Apparently the number of HMXBs normalized by the mass of the galaxies is much higher in the SMC than in our Galaxy and the LMC. Unusually, all but one of the X-ray binaries so far discovered in the MCs are HMXBs. The number ratio of HMXBs to LMXBs exhibits a striking difference between the MCs and our Galaxy, as is already pointed out by Schmidtke et al. (1999). Such a high density of HMXB population must provide clues about the star formation rates in the SMC, since HMXBs could be an indicator of star formation rate (Grimm et al. 2003) while LMXBs are generally considered to comprise an older population than HMXBs. The MCs should be very active in massive star formation, which is consistent with the results that MCs have experienced encounters with the Galaxy in past and the encounters likely triggered bursts of star formation (see e.g. Yoshizawa \& Noguchi 2003).

In contrast to the large number of HMXBs in the MCs, it is very unusual that there are only two black hole candidates in the LMC and no black hole X-ray binaries in the SMC, while more than 40 black hole candidates were found in the Galaxy. However, Zhang, Li \& Wang (2004) found that it is very difficult to detect black hole/Be systems, due to the efficient truncation of the Be circumstellar disk by black holes. 
Table 1. Source populations in the MCs and the Galaxy

\begin{tabular}{lcccccc}
\hline Galaxy & \multicolumn{3}{c}{ HMXBs } & \multicolumn{2}{c}{ LMXBs } & Ref. \\
\hline & Total & Pulsar & BHC & Total & BHC \\
\hline SMC & 92 & 47 & 0 & 0 & 0 & Liu et al. 2005 \\
\hline LMC & 36 & 7 & 2 & 1 & 0 & Liu et al. 2005 \\
\hline Galaxy & $\sim 110$ & 57 & $2(?)$ & $\sim 200$ & $34(?)$ & Liu et al. 2000,2001 \\
\hline
\end{tabular}

\section{Pulse period distribution of HMXBs}

A simple scaling based on the relative masses of the MCs and the Galaxy predicts only 2 pulsars in the SMC and 20 in the LMC. However, currently 49 X-ray pulsars are known in the SMC (Coe et al. 2005), and 7 in the LMC. All pulsars except 2 in the SMC are most likely in HMXB systems as suggested by their X-ray properties. Fig. 1 shows the pulse period distributions of the X-ray pulsars in the MCs and our Galaxy. On average, the pulse periods in the SMC are slightly shorter than those in the Galaxy. Since the pulsars in the SMC are likely much younger than those in the Galaxy, it is likely that most of the pulsars in Be/X-ray binaries are not spin-up.

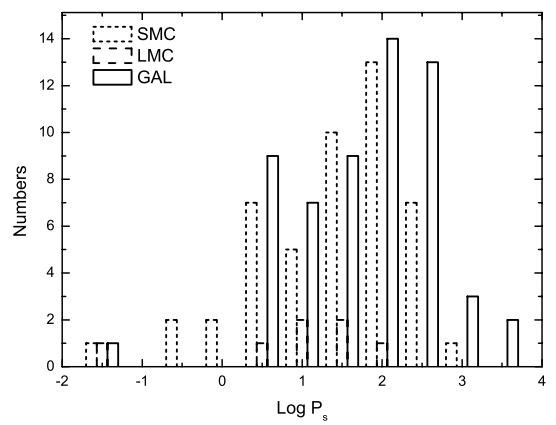

Figure 1. A comparison of the pulse period distributions of the X-ray pulsars in the 3 galaxies.

\section{Acknowledgements}

I thank the support from NSFC under Grants No. 10173026 and No. 10433030.

\section{References}

Coe, M. J. et al. 2005, MNRAS, 356, 502

Grimm, H.-J., Gilfanov, M., \& Sunyaev, R. 2003, MNRAS, 339, 793

Liu, Q. Z., van Paradijs, J. \& van den Heuvel, E. P. J. 2000, A\&AS, 147, 25

Liu, Q. Z., van Paradijs, J. \& van den Heuvel, E. P. J. 2001, A\&A, 368, 1021

Liu, Q. Z., van Paradijs, J. \& van den Heuvel, E. P. J. 2005, A\&A, in press

Schmidtke, P. C., Cowley, A. P., Crane, J. D. et al. 1999, AJ, 117, 927

Yoshizawa, A. M. \& Noguchi, M. 2003, MNRAS, 339, 1135

Zhang, F., Li, X.-D. \& Wang, Z.-R. 2004, ApJ, 603, 663 